compared with 474 children with other types of epilepsy, and 2196 children with febrile seizures, admitted with neurologic disorders at age 1 month to 2 years to 12 Danish pediatric departments during 1967-68 and 1972-73. There was a family history of seizures in $14 \%$ of children with infantile spasms, compared to $29 \%$ in children with other epilepsies, $26 \%$ in those with febrile seizures, and $5 \%$ in children admitted for CNS infections. A family history of seizures increased the risk for infantile spasms threefold, but only in the cryptogenic cases that made up $50 \%$ of the group. Neonatal hypoxia, neonatal seizures, and CNS malformations were much stronger predictors of infantile spasms than genetic factors. The relatively low incidence of organic cases in this study was explained by the lack of available brain imaging. (Rantala $\mathrm{H}$, Shields WD et al. Risk factors of infantile spasms compared with other seizures in children under 2 years of age. Epilepsia April 1996;37:362-366). (Reprints: Dr H Rantala, Department of Pediatrics, University of Oulu, FIN-90220 Oulu 22, Finland).

COMMENT. A family history of seizures contributes to a 3-fold risk for infantile spasms of the cryptogenic variety. Organic brain disorders and neurologic abnormalities play a much stronger role as precursors of infantile spasms. Neonatal seizures are particularly predictive of possible occurrence of infantile spasms.

Long-term outcome of West syndrome was studied in 214 children with a history of infantile spasms followed for 20-35 years or until death (31\%) at the Children's Hospital, University of Helsinki, Finland. (Riikonen R. Epilepsia April 1996;37:367-372). Infection was the most frequent cause of death (31 of 67 patients). Eight children who died during treatment with large doses of ACTH had enlarged adrenal glands and hypertrophic cardiomyopathy. Among survivors, intelligence was normal or near normal in 24\%. Factors predictive of a good prognosis included a crytogenic etiology, normal development before onset of spasms, and a good response to ACTH. Focal abnormalities in the EEG were not necessarily indicative of a poor prognosis.

Treatment with high-dose ACTH $(150 \mathrm{U} / \mathrm{m} 2 /$ day $)$ was superior to prednisone (2 $\mathrm{mg} / \mathrm{kg} /$ day) in suppressing clinical spasms and hypsarrhythmia in the EEG in a prospective, randomized, blinded study of 29 patients (22 symptomatic and 7 cryptogenic etiologies) treated for 2 week periods at the University of Southern California, Los Angeles (Baram TZ et al. Pediatrics March 1996;97:375-379). Of 15 patients randomized to ACTH, 13 (87\%) responded, compared to 4 (29\%) of 14 given prednisone.

\title{
LOCALIZING VALUE OF CLINICAL SEIZURE PATTERNS
}

The value of ictal clinical manifestations in differentiating frontal and temporal lobe partial epilepsies was determined in a prospective study of 252 patients selected according to imaging, EEG, and focal clinical patterns from records at the National Hospital for Neurology and Neurosurgery, , London, UK. Cluster analysis gave 14 distinctive clinical types of patterns, but these had limited localizing value with the exception of perirolandic seizures. Two seizure types with prominent, early motor manifestations, especially version and posturing, were associated with frontal lobe abnormalities. Very high seizure frequencies were seen more often with frontal lesions. Seizures associated with temporal lobe lesions were characterized by absences and those with subjective onsets such as fear and oroalimentary automatisms. Location of interictal EEG spikes and ictal EEG onsets were generally consistent with lesion sites. Relatively few seizures could be localized reliably on clinical grounds. ( Manford M et al. An analysis of clinical seizure patterns and their 
localizing value in frontal and temporal lobe epilepsies. Brain Feb 1996;119:1740). (Respond: Dr M Manford, Wessex Neurological Centre, Southampton General Hospital, Tremona Road, Southampton SO16 6YD, UK).

COMMENT. The ILAE classification of seizure types based on electroclinical criteria may be justified for well-defined syndromes, eg benign epilepsy of childhood with centrotemporal spikes, but in the differentiation of frontal and temporal lobe partial epilepsies in adults there is significant clinical/pathological overlap. The authors argue in favor of an anatomicalpathological definition of fronto-temporal seizures, and they disagree with the ILAE classification of 7 different localizable seizure types within the frontal lobes based on electroclinical manifestations. Neuroimaging appears to be the only reliable method of lesion localization for frontal and temporal lobe partial epilepsies.

Magnetoencephalographic analysis of rolandic discharges in benign childhood epilepsy is reported from Kyushi University, Fukuoka, Japan. (Minami T et al. Ann Neurol March 1996;39:326-334). Dipole methods of MEG allow more precise location and quantification of electrically active brain regions compared to the EEG, and the recorded signal reflects intracellular rather than extracellular current flow. Equivalent current dipoles (ECDs) of prominent negative sharp waves of rolandic discharges appeared as tangential dipoles in the rolandic region and showed a limited localization compared with other components. ECDs of preceding small positive waves, positive waves following negative sharp waves, and negative slow waves were located close to negative sharp waves. ECDs of rolandic discharges were localized to the origin of somatosensory evoked magnetic field stimulated at the lower lip on a reconstructed three-dimensional MR image. Rolandic discharges are generated by a similar mechanism to that for the somatosensory evoked responses.

\section{KETOGENIC DIET MECHANISM OF ACTION}

The intracellular $\mathrm{pH}$ of the cerebral cortex was studied by the neutral red method in 15 adult rats maintained on ketogenic and control diets for 5-6 weeks. Rats fed the ketogenic diet had more than a 10-fold increase in plasma ketones, but no significant differences in cerebral $\mathrm{pH}$ or in cerebral metabolites and GABA levels were noted. The antiepileptic effect of the ketogenic diet was probably not mediated by cerebral acidosis or changes in cerebral GABA levels. (Al-Mudallal AS, Harik SI et al. Diet-induced ketosis does not cause cerebral acidosis. Epilepsia April 1996;37:258-261). (Reprints: Dr SI Harik, Department of Neurology, University of Arkansas, 4301 W Markham, Slot 500, Little Rock, AR 72205).

COMMENT. The ketogenic diet used in the treatment of some forms of epilepsy in childhood was originally introduced at the Mayo Clinic (Wilder RM. Mayo Clin Bull 1921;2:307), not at Johns Hopkins University, as some recent media publicity would have us believe. Furthermore, some of the earlier work relating to the mechanism of action of the ketogenic diet, not cited in the above paper, also originated at the Mayo Clinic (Millichap JG, Jones JD, Rudis BP. Mechanism of anticonvulsant action of ketogenic diet. Amer I Dis Child 1962;104:506, and 1964;107:593-604). Seizure susceptibility was not modified by a fat diet in normal animals, but an anticonvulsant effect was demonstrated in mice with a seizure threshold lowered by water intoxication and hypoelectrolytemia. In animals and in patients with absence seizures, the 